To appear in Phys. Rev. D -Brief Reports

TRI-PP-96-13

hep-ph/9608303

\title{
A NOTE ON THE EXTERNAL-FIELD METHOD IN QCD SUM RULES
}

\author{
Xuemin Jin* \\ TRIUMF, 4004 Wesbrook Mall, Vancouver, B.C. V6T 2A3, Canada \\ and Institute for Nuclear Theory, University of Washington, Seattle, WA 98195, USA
}

\begin{abstract}
The external-field method has been used extensively in the QCD sum-rule approach to explore various hadron static properties. In the traditional formalism of this method, the transitions from the ground state hadron to excited states are not exponentially suppressed relative to the ground state term and thus contaminate the ground state hadron property to be extracted. In this paper, we suggest a modified formalism, in which the transition terms are exponentially suppressed relative to the ground state term. As such, the pole plus continuum spectral model, traditionally invoked in QCD sum-rule approach, can be adopted. Thus, this modified formalism has potential to improve the predictability and reliability of external-field sum-rule calculations, which is illustrated in an explicit example.
\end{abstract}

PACS numbers: 12.38.Lg, 11.55.Hx

*Present address: Center for Theoretical Physics, Laboratory for Nuclear Science and Department of Physics, Massachusetts Institute of Technology, Cambridge, Massachusetts 02139, USA 
The QCD sum-rule approach [1] is a useful tool of extracting qualitative and quantitative information about hadron properties. One of the extensions of this approach made by Ioffe and Smilga [2] for external-field problems enables one to study hadron matrix elements of various currents and corresponding hadron static properties [2] 18].

In the traditional external-field QCD sum-rule formalism, the contributions of transitions from the ground state hadron to excited states are not exponentially suppressed (after Borel transformation) relative to the ground state contribution which contains the ground state property to be determined. There are, in general, infinitely many transition terms as there are infinitely many excited states, and such terms should be retained in the calculations. The usual approximation adopted in the literature is to introduce a new unknown phenomenological parameter (independent of Borel mass) accounting for the sum over the contributions from all the transitions between the ground state and the excited states. This new parameter is extracted from the sum rules, along with the ground state property. The isolation of the ground state property relies on that the polynomial (in Borel mass) behavior of the ground state signal is different from those of the transition terms. While this approximation has been used in earlier studies, it has been noticed recently that the parameter representing the transitions, in general, should be dependent on the Borel mass, which can have a sizable impact on the extracted ground state properties [19, 17].

In this paper, we point out that a modified form for the linear response of hadron correlation function to the external field can lead to exponential suppression of the transition terms relative to the ground state term. As such, the pole plus continuum spectral model, usually adopted in QCD sum-rule approach, can be adopted. This formalism has potential to improve the predictability and reliability of external-field sum-rule calculations. A comparison to the traditional formalism is illustrated in an explicit example.

The external-field method in QCD sum rules starts from a correlation function of hadron interpolating fields in the presence of a constant external field [2] 18

$$
\Pi(S, q) \equiv i \int d^{4} x e^{i q \cdot x}\left\langle 0\left|T\left[\eta_{H}(x) \bar{\eta}_{H}(0)\right]\right| 0\right\rangle_{S},
$$

where $S$ denotes the external field and $\eta_{H}$ is a hadron interpolating field constructed from local QCD quark and gluon operators, carrying the quantum numbers of the hadron under investigation. The presence of the external field implies that $\Pi(S, q)$ is evaluated with an additional term

$$
\Delta \mathcal{L} \equiv-S \cdot J,
$$

added to the usual QCD Lagrangian, where $J$ denotes the current of interest. Hereafter, all possible Lorentz indices of $J$ and $S$ will be suppressed.

To proceed, one usually expands Eq. (11) to first order in the external field

$$
\Pi(S, q)=\Pi_{0}(q)+\Pi_{1}(q) \cdot S+\cdots,
$$

where $\Pi_{0}(q)$ is the correlation function in the absence of the external field, which gives rise to the usual sum rules for hadron masses. One then focuses only on the linear response term $\Pi_{1}(q)$ which can be expressed as 


$$
\Pi_{1}(q)=\int d^{4} x e^{i q \cdot x}\left\langle 0\left|T\left\{\eta_{H}(x)\left[\int d^{4} y J(y)\right] \bar{\eta}_{H}(0)\right\}\right| 0\right\rangle .
$$

The QCD representation of $\Pi_{1}(q)$, in terms of an operator product expansion (OPE), can be obtained by treating the external field perturbatively and then identifying the terms linear in $S$. The phenomenological representation for $\Pi_{1}(q)$ can be obtained by expanding $\Pi_{1}(q)$ in terms of physical hadron intermediate states or by invoking a dispersion relation. Here we follow the former to keep our discussion succinct. In a discrete-state approximation (i.e., neglecting the widths of hadron states, which is usually assumed in the QCD sum-rule approach [1]), the phenomenological representation can be expressed as

$$
\begin{aligned}
\Pi_{1}\left(q^{2}\right) & \sim\left\langle 0|\eta| h_{0}\right\rangle\left\langle h_{0}|J| h_{0}\right\rangle\left\langle h_{0}\left|\bar{\eta}_{H}\right| 0\right\rangle \frac{1}{\left(M_{0}^{2}-q^{2}\right)^{2}} \\
& +\sum_{i \neq 0}\left[\left\langle 0|\eta| h_{0}\right\rangle\left\langle h_{0}|J| h_{i}\right\rangle\left\langle h_{i}\left|\bar{\eta}_{H}\right| 0\right\rangle+\text { h.c }\right] \frac{1}{\left(M_{0}^{2}-q^{2}\right)\left(M_{i}^{2}-q^{2}\right)} \\
& + \text { terms involving only excited states }
\end{aligned}
$$

where $\left|h_{0}\right\rangle$ and $\left|h_{i}\right\rangle$ denote the ground and excited states with mass $M_{0}$ and $M_{i}$, respectively. The first term contains the hadron matrix element, $\left\langle h_{0}|J| h_{0}\right\rangle$, of interest, the second term is the transition term from ground state to excited states usually discussed in the literature, and the rest involves only the excited states. After a usual Borel transformation [1], one finds

$$
\begin{aligned}
\Pi_{1}\left(M^{2}\right) & \sim \frac{\lambda_{0}^{2}}{M^{2}}\left\langle h_{0}|J| h_{0}\right\rangle e^{-M_{0}^{2} / M^{2}}+\sum_{i \neq 0}\left[\frac{\lambda_{0} \lambda_{i}\left\langle h_{0}|J| h_{i}\right\rangle}{M_{i}^{2}-M_{0}^{2}}\left(1-e^{-\left(M_{i}^{2}-M_{0}^{2}\right) / M^{2}}\right)+\text { h.c }\right] e^{-M_{0}^{2} / M^{2}} \\
& + \text { exponentially suppressed terms }
\end{aligned}
$$

where $\lambda_{0}$ and $\lambda_{i}$ denote the coupling strengths of the hadron interpolating field to the ground and excited states, respectively, and $M$ is the Borel mass.

It can be seen clearly that the second term in Eq. (6) is not exponentially damped as compared to the first term. This has been stressed constantly in the literature [2]18]. The usual strategy is to approximate the second (transition) term as $A e^{-M_{0}^{2} / M^{2}}$ with $A$ a constant to be extracted from the sum rule along with the hadron matrix element of interest. However, one notices that $A$, in general, is a complicated function of the Borel mass $M^{2}$. The neglect of such a Borel mass dependence will generate errors in the extracted hadron matrix element (see for example Refs. [19,17]).

To improve this situation, we suggest to use the combination $\left(M_{0}^{2}-q^{2}\right) \Pi_{1}\left(q^{2}\right)$, instead of $\Pi_{1}\left(q^{2}\right)$ alone. The corresponding sum rule for this combination has the form

$$
\begin{aligned}
\widetilde{\Pi}_{1}\left(M^{2}\right) & \sim \lambda_{0}^{2}\left\langle h_{0}|J| h_{0}\right\rangle e^{-M_{0}^{2} / M^{2}}+\sum_{i \neq 0}\left[\lambda_{0} \lambda_{i}\left\langle h_{0}|J| h_{i}\right\rangle+\text { h.c }\right] e^{-M_{i}^{2} / M^{2}} \\
& + \text { exponentially suppressed terms } \\
& \equiv \lambda_{0}^{2}\left\langle h_{0}|J| h_{0}\right\rangle e^{-M_{0}^{2} / M^{2}}+\sum_{i \neq 0} A_{i} e^{-M_{i}^{2} / M^{2}}
\end{aligned}
$$


The transitions from ground state to excited states (second term) are now exponentially suppressed with respect to the ground state (first) term. This indicates that one may implement the traditional pole plus continuum model for the spectral ansatz of $\widetilde{\Pi}_{1}$. That is, one may model $\sum_{i \neq 0} A_{i} e^{-M_{i}^{2} / M^{2}}$ in terms of the perturbative evaluation of $\Pi_{1}$, starting from an effective threshold. As such, one no longer needs to introduce any phenomenological parameter to represent the transitions from the ground state to excited states as their contributions have been included in the continuum model. Therefore, this new formalism has potential to improve the predictability and reliability of the external-field method.

As an example of illustration, we consider the proton matrix element of isovector-scalar current, $H \equiv\langle p|\bar{u} u-\bar{d} d| p\rangle / 2 M_{p}$. This matrix element has been studied in Ref. [16] within the traditional external-field QCD sum-rule method (see also Ref. [17]). Here, our purpose is only to compare the new formalism suggested above with the traditional formalism. Thus, we shall adopt the same proton interpolating field and notations as used in Ref. [16].

In the traditional formalism, the sum rule used to extract $H$ is given by [16]

$$
\begin{aligned}
2 H \widetilde{\lambda}_{p}^{2} & \frac{M_{p}}{M^{2}} e^{-M_{p}^{2} / M^{2}}+\widetilde{A} e^{-M_{p}^{2} / M^{2}} \\
& =-2 a M^{2} E_{0} L^{-4 / 9}+\frac{8}{3} \chi a^{2} L^{4 / 9}+\frac{1}{3} m_{0}^{2} a L^{-8 / 9}-\frac{2 \chi}{3 M^{2}} a^{2} m_{0}^{2} L^{-2 / 27},
\end{aligned}
$$

where $M_{p}$ is the proton mass, $L=\ln \left(M^{2} / \Lambda_{\mathrm{QCD}}^{2}\right) / \ln \left(\mu^{2} / \Lambda_{\mathrm{QCD}}^{2}\right)$, and $E_{0}=1-e^{-s_{0} / M^{2}}$ with $s_{0}$ the continuum threshold. The values of $a, m_{0}^{2}, \Lambda_{\mathrm{QCD}}$, and $\mu$ can be found in Ref. [16]. In the new formalism of Eq. (8), the sum rule becomes

$$
\begin{aligned}
2 H \widetilde{\lambda}_{p}^{2} M_{p} e^{-M_{p}^{2} / M^{2}}= & -2 a\left(M_{p}^{2} E_{0}-M^{2} E_{1}\right) M^{2} L^{-4 / 9}+\frac{8}{3} \chi a^{2} M_{p}^{2} L^{4 / 9} \\
& +\frac{1}{3} m_{0}^{2} a M_{p}^{2} L^{-8 / 9}-\frac{2 \chi}{3}\left(1+\frac{M_{p}^{2}}{M^{2}}\right) a^{2} m_{0}^{2} L^{-2 / 27},
\end{aligned}
$$

where $E_{1}=1-\left(1+s_{0} / M^{2}\right) e^{-s_{0} / M^{2}}$. In Eq. (9) , the transition term from the proton to excited states is not exponentially suppressed relative to the ground state term and is approximated as a constant phenomenological parameter $\widetilde{A}$ multiplied by $e^{-M_{p}^{2} / M^{2}}$. This parameter is to be determined from the sum rule along with the matrix element $H$ of interest. On the other hand, there is no such parameter in Eq. (10) as the transition term is exponentially suppressed with respect to the ground state term [see Eq. (B)] and its contribution is included in the continuum model.

To extract $H$ from the above sum rules, we follow the numerical optimization procedure adopted in Refs. [16, 17]. For definiteness, we take for $\chi$ the value of $\chi=2.2 \mathrm{GeV}^{-1}$ and the same Borel window as used in Ref. [16]. In the traditional method, the continuum threshold $s_{0}$ is taken to be the same as that in the mass sum rules and $H$ and $\widetilde{A}$ are extracted from the sum rule [2 [16]. We use $\widetilde{\lambda}_{p}^{2} \simeq 1.85 \mathrm{GeV}^{6}$ and $s_{0} \simeq 2.14 \mathrm{GeV}^{2}$ [17], which are slightly different from those used in Ref. [16]. In the new formalism, however, we extract both $H$ and the continuum threshold $s_{0}$ from the sum rule as the contribution of the transitions is effectively included in the continuum model. The predictions turn out to be 


$$
\begin{array}{ll}
H \simeq 0.60 & \text { [from Eq. (9)] } \\
H \simeq 0.97 & \text { [from Eq. (10)] } .
\end{array}
$$

So, the two sum rules give very different predictions for the matrix element $H$. Which one is more reliable?

We have demonstrated in Eq. (6) that the parameter $\widetilde{A}$ in the traditional formalism should be a complicated function of the Borel mass. Ignoring this Borel mass dependence will alter the curvature of the phenomenological side of the sum rule and hence generate errors in sum-rule predictions. This, for the example under consideration, has been discussed in Ref. [17] (see also Ref. [19]). In particular, a complete form for the phenomenological side, which must be invoked in the traditional formalism, has been derived, and the corresponding sum rule takes the form [17]

$$
\begin{aligned}
2 H \widetilde{\lambda}_{p}^{2} & \frac{M_{p}}{M^{2}} e^{-M_{p}^{2} / M^{2}}+\widetilde{A} e^{-M_{p}^{2} / M^{2}}+\widetilde{B} \frac{s_{0}^{2}}{2} L^{-8 / 9} e^{-s_{0} / M^{2}} \\
& =-2 a M^{2} E_{0} L^{-4 / 9}+\frac{8}{3} \chi a^{2} L^{4 / 9}+\frac{1}{3} m_{0}^{2} a L^{-8 / 9}-\frac{2 \chi}{3 M^{2}} a^{2} m_{0}^{2} L^{-2 / 27} .
\end{aligned}
$$

It is the last term on the left-hand side which is ignored in the traditional formalism Eq. (9). This term is Borel mass dependent and has impact on the prediction for $H$. There are three parameters $H, \widetilde{A}$, and $\widetilde{B}$ to be extracted from this sum rule, and $s_{0}$ is to be fixed at its value in the mass sum rules. Using the same numerical procedure, we find

$$
H \simeq 0.98 \quad \text { [from Eq. (13)] }
$$

which is very close to the result from the new formalism [Eq. (12)]. This indicates that the traditional treatment of the transition term has serious drawbacks arising from the approximation adopted, which can lead to large errors in the sum-rule predictions for the ground state matrix element. The new formalism, on the other hand, overcomes these drawbacks and greatly improves the reliability of the sum-rule predictions.

One notices that the contribution of continuum in Eq. (10) differs from that in Eq. (9). This rises the question as to whether the change in the continuum contribution will affect the accuracy of the new formalism. We note that in the new formalism the contributions from the transitions are included in the continuum model. It is thus fair to make a comparison between the relative contribution of the continuum to the total phenomenological side in the new formalism and the relative contribution of the transition term plus continuum to the total phenomenological side in the traditional formalism. The result is shown in Fig. 1. We see that the contribution of the continuum in the new formalism is smaller than that of the continuum plus transitions in the traditional formalism in most of the Borel window. This can also be seen from that a term of the continuum contribution in the traditional formalism becomes two terms in the new formalism which tend to cancel each other[see Eq. (9) and (10)]. Thus, we conclude that the change in the continuum contribution does not affect the accuracy of the sum-rule prediction in the new formalism.

Since the result from the new formalism is very similar to that from the traditional formalism with a complete form for the phenomenological representation [Eq. (13)], one 


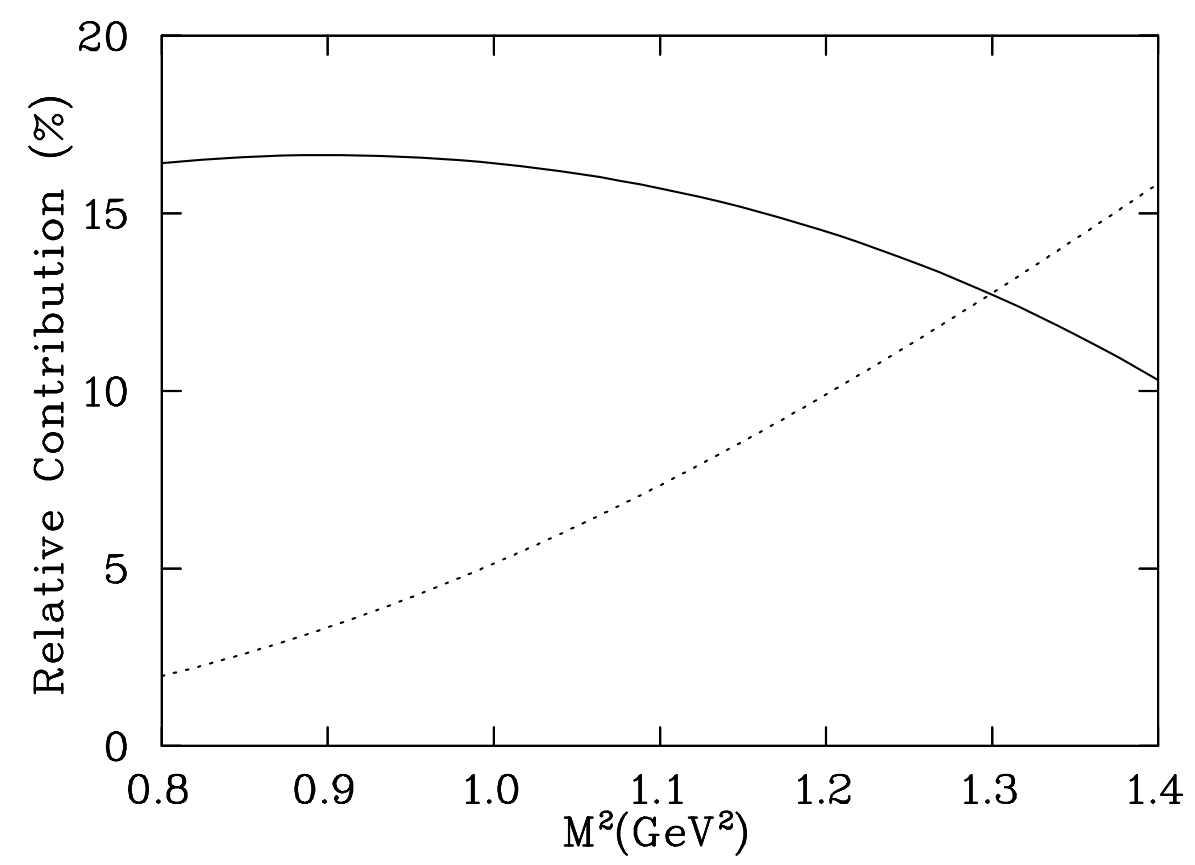

FIG. 1. The relative contribution (solid curve) of the transition term plus continuum to the total phenomenological side in the traditional formalism Eq. (9) compared to that (dotted curve) of the continuum to the total phenomenological side in the new formalism Eq. (10).

may wonder the advantages of the new formalism. An obvious one is that the usual pole plus continuum model can be used in the new formalism, which reduces the number of phenomenological parameters to be extracted from the sum rules [e.g., $H$ and $s_{0}$ in the new formalism vs. $H, \widetilde{A}$, and $\widetilde{B}$ in Eq. (13)]. A more important advantage is that the new formalism can be applied to external-field problems with any kind of external fields (vector, tensor, etc.) while a complete form for the phenomenological representation needed in obtaining Eq. (13) cannot be derived explicitly in the case of non-scalar external fields.

The external-field method used in QCD sum-rule calculations concerns only the linear response term $\Pi_{1}(q)$ of the correlation function, which is actually a three-point function [Eq. (田)]. So one may evaluate $\Pi_{1}(q)$ by following the usual external-field method or by applying the OPE directly to the time-ordered operator product in $\Pi_{1}(q)$. The latter was initiated by Balitsky and Yung [20] and followed by others [21,22]. The resulting sum rules in these two apparently different approaches must be identical. The apparent differences are only in the organization of the terms on the QCD sides of the sum rules. However, the calculations in the external-field method are usually simpler and more transparent than in the direct three-point function calculations.

It is interesting to note that Balitsky and Yung [20] have proposed a procedure similar to the one discussed in the present paper. Such procedure has been adopted in later QCD sumrule calculations based directly on three-point functions [21,22], but not in the external-field method. However, the authors of Refs. [20,21] stated that the transitions from the ground state to the excited states are eliminated or canceled out in the sum rule for $\widetilde{\Pi}_{1}$. This statement is obviously not true. The transitions from the ground state to the excited states 
do contribute to the sum rule, and their contributions are included in the continuum model. We also note that in Ref. [20 22] the continuum threshold in the sum rule for $\widetilde{\Pi}_{1}(q)$ has been assumed to be the same as the one in the mass sum rules. This assumption, as shown above, is unjustified. Using the same threshold in these two cases will introduce artificial bias to the extracted hadron properties.

As emphasized in Ref. [17], it is the product of $\lambda_{H}^{2}$ and the hadron matrix element of interest that usually appears in the sum rules for $\widetilde{\Pi}_{1}(q)$. One then needs a good knowledge of $\lambda_{H}^{2}$ in order to extract the interested hadron matrix elements cleanly. This means that uncertainties associated with $\lambda_{H}^{2}$ may give rise to additional uncertainties in the determination of the hadron matrix elements. This is a general drawback of the sum rules for $\Pi_{1}(q)$. This drawback can only be sidestepped if the external field is treated non-perturbatively. The reader is referred to Ref. [23] for more discussions on this point.

In summary, we have pointed out that a modified form for the linear response of hadron correlation function to the external field, concerned in the external-field method in QCD sum rules, can lead to exponential suppression of the transitions from ground state to excited states relative to the ground state term and hence reduce the transition contamination to the ground state property. This approach allows one to use the traditional pole plus continuum spectral model and thus has potential to improve the predictability and reliability of externalfield sum-rule calculations, which has been illustrated in an explicit example. Our hope is that this new formalism of the external-field method will generate new interest in revealing hadron static properties via the external-field QCD sum-rule method.

I would like to thank the Institute for Nuclear Theory at the University of Washington for its hospitality and the US Department of Energy for partial support during the completion of this work. I am indebted to Derek Leinweber for insightful conversations. The support from the Natural Sciences and Engineering Research Council of Canada is gratefully acknowledged. 


\section{REFERENCES}

[1] M. A. Shifman, A. I. Vainshtein, and V. I. Zakharov, Nucl. Phys. B147, 385 (1979); B147, 448 (1979).

[2] B. L. Ioffe and A. V. Smilga, Pis'ma ZH. Eksp. Teor. Fiz. 37, 250 (1983) [JETP Lett. 37, 298 (1983)]; Nucl. Phys. B232, 109 (1984).

[3] B. L. Ioffe and A. V. Smilga, Phys. Lett. 133B, 436 (1983).

[4] C. B. Chiu, J. Pasupathy, and S. L. Wilson, Phys. Rev. D 33, 1961 (1986).

[5] J. Pasupathy, J.P. Singh, S.L. Wilson, and C.B. Chiu, Phys. Rev. D 36, 1442 (1987).

[6] C.B. Chiu, S.L. Wilson, J. Pasupathy, and J.P. Singh, Phys. Rev. D 36, 1553 (1987).

[7] V. M. Belyaev and Ya. I. Kogan, Pis'ma ZH. Eksp. Teor. Fiz. 37, 611 (1983) [JETP Lett. 37, 730 (1983)]; Phys. Lett. B136, 273 (1984).

[8] V. M. Belyaev, B. L. Ioffe, and Ya. I. Kogan, Phys. Lett. 151B, 290 (1985).

[9] C. B. Chiu, J. Pasupathy, and S. L. Wilson, Phys. Rev. D 32, 1786 (1985).

[10] S. Gupta, M. V. N. Murthy, and J. Pasupathy, Phys. Rev. D 39, 22547 (1989).

[11] I. I. Balitsky, V. M. Braun, and A. V. Kolesnichenko, Phys. Lett. B242, 245 (1990).

[12] E. M. Henley, W.-Y. P. Hwang, and L. S. Kisslinger, Phys. Rev. D 46, 431 (1992).

[13] B. L. Ioffe and A. Yu. Khodzhamirian, Sov. J. Nucl. Phys. 55, 1701 (1992).

[14] V. M. Belyaev and B. Yu. Blok, Z. Phys. C 30, 279 (1986); Phys. Lett. 167B, 99 (1986).

[15] X. Jin, M. Nielsen, and J. Pasupathy, Phys. Lett. B314, 163 (1993).

[16] X. Jin, M. Nielsen, and J. Pasupathy, Phys. Rev. D 51, 3688 (1995).

[17] X. Jin, Phys. Rev. D 52, 2964 (1995).

[18] X. Jin, TRIUMF report No. TRI-PP-96-2, hep-ph/9602298, (1996).

[19] B. L. Ioffe, Phys. Atom. Nucl. 58, 1408 (1995).

[20] I. I. Balitsky and A. V. Yung, Phys. Lett. 129B, 328 (1983).

[21] V. M. Braun and A. V. Kolesnichenko, Nucl. Phys. B283, 723 (1987).

[22] H. He and X. Ji, Phys. Rev. D 52, 2960 (1995).

[23] M. Burkardt, D. B. Leinweber, and X. Jin, Phys. Lett. B385, 52 (1996). 